(Aus der Dermatologischen Universitätsklinik Breslau [Direktor: Geheimrat Jadassohn].)

\title{
Die Lungentuberkulose als Komplikation der Tuberkuloderme.
}

\author{
Von \\ Dr. med. Hans Martenstein. \\ Assistenzarzt der Klinik.
}

Mit 2 Textabbildungen.

Die Frage, wie oft Patienten mit Hauttuberkulose auch an innerer Tuberkulose erkrankt sind, ist bereits von vielen Autoren bearbeitet worden. Jadassoh $\mathrm{n}$ zitiert in seiner Abhandlung über ,die Tuberkulose der Haut" im Handbuch von Mrace k eine ganze Reihe von statistischen Zusammenstellungen auf diesem Gebiete. Ein näheres Eingehen auf die in ihnen angegebenen Zahlen dürfte wohl unnötig sein, da sie in erheblichem Maße voneinander abweichen. Jadassohn ${ }^{\mathbf{1}}$ ) führt dies, außer auf die Möglichkeit einer Verschiedenheit des Materials, auf Differenzen in der Bewertung einzelner physikalischer Symptome für die Diagnose einer beginnenden tuberkulösen Lungenaffektion zurück. Nur die beiden ausgedehntesten Statistiken ergeben ein gut übereinstimmendes Resultat, was sich wohl durch einen Ausgleich der Fehldiagnosen bei nicht deutlich ausgesprochenen Lungenbefunden erklären läßt. Es sind dies die Zusammenstellungen von Leloir²) über 312 Lupusfälle und die von Grouven $n^{3}$ ) über 585 Fälle von Lupus vulgaris und Skrofuloderm. Nach Leloir findet sich in $31,4 \%$ eine Komplikation mit Lungentuberkulose, nach Grouven in 29,9\%. Aus den Angaben der Statistiken geht außerdem hervor, daß das Hauptkontingent der Hauttuberkulosen in ihnen von der Tuberculosis luposa gestellt wird, andererseits, daß die viscerale Tuberkulose ausschließlich die Lungen betrifft. Die gleiche Tatsache ergibt die neueste Untersuchung über ein größeres Material auf diesem Gebiet, die Forchham mer ${ }^{4}$ ) im Jahre 1908 aus Finsens medizinske Lysinstitut in Kopenhagen veröffentlicht

1) Jadassohn, 1. c. S. 303 .

2) Leloir, ebenda S. 304.

3) Grouven, Anderw. Tub. bei Lup. u. Skrofuloderm., Beitr. z. Klinik d. Tuberkulose 1, 159.

4) Forchhammer, Über Lungentuberkulose als Todesursache bei Lupus vulgaris. Dieses Archiv 92, 1. 1908. 
hat. Allerdings lassen sich Forch ha m mers Daten nicht ohne weiteres mit den Angaben der oben angeführten Statistiken vergleichen, da er als Hauptziel die Feststellung der Lungentuberkulose als Todesursache bei Lupus valgaris verfolgt. Er hat im Verlaufe von $10 \mathrm{Jahren} 1190 \mathrm{Lu}$ pusfälle aus ganz Dänemark beobachtet und gefunden, daß von ihnen 81 an tuberkulösen Krankheiten starben, darunter 74, also etwas mehr als $6 \%$ der Gesamtzahl, an Phthisis pulmonum. Ein Schluß auf die Zahl der bei seinen Patienten außerdem bestehenden tuberkulösen Lungenaffektionen, die während der Beobachtungszeit nicht zum Exitus kamen, läßt sich nicht ziehen. Da, soweit mir bekannt, größere Zusammenstellungen über das hier besprochene Thema seitdem nicht publiziert worden sind, hat man also auf Grund der Literaturangaben mit einer Durchschnittszahl von 30 Fällen einer tuberkulösen Lungenkomplikation unter hundert Lupus-vulgaris-Patienten zu rechnen.

Da der Universitäts-Hautklinik Breslau als Zentralstelle die Lupusfürsorge für den Südosten Deųtschlands angegliedert ist, habe ich in der Lupusabteilung Gelegenheit, ein ziemlich reiches Material von Kranken mit tuberkulösen Erkrankungen der Haut in ihren verschiedenen Variationen zu beobachten. Bei der klinischen Untersuchung der inneren Organe der Patienten fiel mir auf, daß ich bei verhältnismäßig wenig Kranken einen positiven Lungenbefund erheben konnte. Jedenfalls schien mir ihre Zahl erheblich geringer zu sein, als die, die nach dem oben angeführten Verhältnis angenommen werden mußte. Eine mangelhafte Untersuchungstechnik als alleinige Erklärung für meine abweichenden Ergebnisse glaubte ich nicht annehmen zu dürfen, da ich früher längere Zeit hindurch zahlreiche Lungenuntersuchungen bei tuberkulösen Affektionen allex Stadien unter Kontrolle des Röntgenschirms bzw. der Röntgenplatte vorzunehmen Gelegenheit hatte. Durch das große Entgegenkommen des Herrn Geheimrat Min kows ki, Direktors der Med. Universitätsklinik Breslau, und seines Assistenten, Herm Schäffer, denen ich auch an dieser Stelle meinen herzlichen Dank dafür aussprechen möchte, ist es möglich gewesen, in den letzten drei Vierteljahren bei hundert unserer an Tuberkulodermen erkrankten Patienten den Lungenbefund röntgenologisch festzustellen. $Z_{W}$ ar ist die $Z$ ahl der von uns untersuchten Kranken erheblich kleiner, als in den drei oben angeführten Statistiken, doch glaube ich, daß das Ergebnis unserer Zusammenstellung mindestens den gleichen Anspruch in bezug auf Verläßlichkeit erheben darf, wie diejenigen von Leloir und Gro u ven. Schaltet doch die Röntgenuntersuchung die von Jadasso hn angegebene. Fehlerquelle der subjektiven Beurteilung von Lungenbefunden so weit als möglich aus. Um diese noch weiter zu verringern, stellte Herr Schäffer seine röntgenologischen Untersuchungen an, ohne Kenntnis des vorher von mir erhobenen klinischen Lungenstatus 
zu haben. Es wurde jeder Patient, soweit äußere Gründe es nicht verhinderten, untersucht ohne Rücksicht auf Behandlungsdauer und Heilerfolg.

Leider muß ich es mir versagen, die einzelnen Krankengeschichten zu veröffentlichen, da sie selbst in Tabellenform zuviel Raum beanspruchen würden. Es sei nur mitgeteilt, daß sie in fünf Gruppen geordnet sind :

A. Auf eine Körperregion beschränkter Lupus vulgaris ohne Erkrankung der Schleimhaut,

B. mit Erkrankung der Schleimhaut.

C. Disseminierter (hämatogener?) Lupus vulgaris.

D. Skrofuloderme.

E. Tuberculosis cutis verrucosa und Übergangsformen.

$\mathrm{Zu} D$ und $E$ ist zu bemerken, daß in diesen Gruppen außer den ganz streng zu ihnen gehörenden Fällen auch die angeführt sind, die wohl mit einer anderen Form der Hauttuberkulose kombiniert sind, bei denen aber die zur Bezeichnung der Gruppe gewählte Art überwiegt. Zum Verständnis der unten wiedergegebenen Tabellen sei noch bemerkt, daß Patienten, die aus Orten mit 5000 Einwohnern und darunter stammen, als Landbewohner gelten. Hielten sich Kranke im Laufe ihres Lebens sowohl in der Stadt, als auch auf dem Lande auf, so ist die längere Aufenthaltsdauer berücksichtigt.

Das Material ist nach folgenden vier Gesichtspunkten einer Durch sicht unterworfen worden:

1. Wie häufig tritt die Lungentuberkulose als Komplikation des Tuberkuloderms auf?

2. In welchem Grade sind die Patienten in den versehiedenen Lebensaltern davon betroffen?

3. Wie verhält sich das Auftreten einer Lungentuberkulose zu dem Alter des tuberkulösen Hautprozesses?

4. In welchem Maße ist die Stadt- bzw. die Landbevölkerung, die an tuberkulösen Hautleiden erkrankt ist, von einer tuberkulösen Lungenaffektion betroffen?

Bei jeder dieser Fragestellungen ist es selbstverständlich von Wichtigkeit, wie sich die verschiedenen Formen der Hauttuberkulose dabei verhalten, wie die Verteilung unter den Geschlechtern ist, andererseits welcher Art der Lungenprozeß ist. Was den letztgenannten Punkt anbetrifft, so habe ich mich nicht an die Einteilung in drei Stadien nach dem Vorgange von Turban gehalten, sondern mich damit begnügt, die positiven Lungenbefunde in zwei Untergruppen zu teilen:

a) Tuberkulöse Lungenprozesse mit Neigung zu bindegewebiger Abheilung, evtl. mit subfebrilen Temperaturen, und ohne katarrhalische Erscheinungen (indurative Lungentuberkulose). 
b) Progressive Lungentuberkulosen mit akuten katarrhalischen usw. Symptomen.

Zu den negativen Lungenbefunden rechne ich, außer den. Fällen, in denen sich überhaupt kein Befund erheben läßt, auch alle diejenigen, in denen die röntgenologische Untersuchung nur eine verstärkte Hiluszeichnung ergibt. Dies ist wohl berechtigt, da man bei Krankenuntersuchungen auf Grund eines nicht tuberkulösen Leidens Hilusverdichtung als einen nicht gerade seltenen Nebenbefund ebenfalls findet. Eine wesentliche Differenz, die zu Schwierigkeiten in der Klassifizierung hätte führen können, hat sich zwischen den erhobenen klinischen und röntgenologischen Lungenbefunden nicht ergeben. Daß der klinische Status keinen abnormen Befund zeigt, wenn die Röntgenuntersuchung lediglich eine Hilusverschattung ergibt, ist ja von vornherein anzunehmen. Besteht eine leichte Schallverkürzung über der Spitze ohne sonstige klinische Symptome bei negativem Röntgenbefund, so ist das perkutorische Ergebnis vernachlässigt, da man dabei am leichtesten einer Täuschung unterworfen ist. Dagegen sind alle Fälle, die bei negativem oder fraglichem klinischen Befund einen verdächtigen röntgenologischen ergeben, als positiv gerechnet (fünf Fälle), trotzdem man gewiß über die Bewertung dieser Fälle verschiedener Meinung sein kann.

Tabelle I

\begin{tabular}{|c|c|c|c|c|c|c|}
\hline Lungenbefund: & 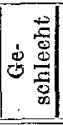 & o.B. & $\begin{array}{c}\text { Hilus- } \\
\text { verdichtung } \\
\text { und Strang- } \\
\text { zeichnung } \\
\end{array}$ & $\begin{array}{l}\text { Lan } \\
\text { tuberk } \\
\text { indu- } \\
\text { rative }\end{array}$ & $\begin{array}{l}\text { llose: } \\
\text { pro- } \\
\text { gress. }\end{array}$ & Summe \\
\hline $\begin{array}{l}\text { A. Lupus vulga ris, auf eine } \\
\text { Körperregion beschränkt, } \\
\text { ohn e Schleimhauterkran- } \\
\text { kung }\end{array}$ & m. & $\begin{array}{l}6 \\
5\end{array}$ & $\begin{array}{l}6 \\
6\end{array}$ & $\begin{array}{l}2 \\
1\end{array}$ & - & $\left.\begin{array}{l}14 \\
12\end{array}\right\}_{26} \mid$ \\
\hline $\begin{array}{l}\text { B. Desgl. mit Schleimhaut- } \\
\text { erkrankung }\end{array}$ & $\begin{array}{c}\mathrm{m} . \\
\mathrm{w} .\end{array}$ & $\begin{array}{l}5 \\
9\end{array}$ & $\begin{array}{l}4 \\
9\end{array}$ & $\begin{array}{l}5 \\
6\end{array}$ & $\begin{array}{l}1 \\
2\end{array}$ & $\left.\left.\begin{array}{l}15 \\
26\end{array}\right\} 41\right\} 80$ \\
\hline $\begin{array}{l}\text { C. Disseminierter Lupus vul- } \\
\text { garis }\end{array}$ & $\begin{array}{c}\text { m. } \\
\text { w. }\end{array}$ & $\begin{array}{l}2 \\
4\end{array}$ & $\begin{array}{l}2 \\
3\end{array}$ & $\overline{1}$ & $\overline{1}$ & $\left.\left.\begin{array}{l}4 \\
9\end{array}\right\} 13\right\} 13$ \\
\hline D. Skrofuloderme & m. & $\begin{array}{l}1 \\
2\end{array}$ & $\begin{array}{l}2 \\
2\end{array}$ & $-\overline{3}$ & - & $\left.\begin{array}{l}3 \\
7\end{array}\right\} 10$ \\
\hline $\begin{array}{l}\text { E. Tuberculosis verr. cutis und } \\
\text { Ubbergangsformen }\end{array}$ & $\begin{array}{c}\mathrm{m} . \\
\mathrm{w} .\end{array}$ & $\underline{2}$ & $\begin{array}{l}4 \\
1\end{array}$ & - & - & 6) 10 \\
\hline Summ & & 36 & $\frac{39}{75}$ & 20 & 5 & 100 \\
\hline
\end{tabular}

1. Wenn bisher von der Lungentuberkulose als Komplikation der Tuberkuloderme gesprochen worden ist, so möchte ich betonen, daB die Frage, welche der beiden Affektionen als die primäre anzusehen ist, 
vollkommen offen gelassen ist, da a priori und nach den Angaben in der Literatur ${ }^{1}{ }^{2}{ }^{3}{ }^{3}$ ) sowohl die Haut, als auch die Lunge primär erkrankt sein kann; ja beide Organe können auch ganz unabhängig voneinander befallen werden. Aus Tabelle I ergibt sich, daß von unseren untersuchten 100 Patienten mit Tuberkuloder men 25 mit einer tuberkulösen Lungenaffektion behaftet sind. Trotz exakterer Untersuchungsmethode und der Hinzurechnung fraglicher Fälle bleibt also für den Südosten Deutschlands der Prozentsatz der Phthisiker unter den Hauttuberkulösen unter der von Leloir und Grouven gefundenen Durchschnittszahl. Dazu kommt noch, daß unter den 25 Lungentuberkulosen 20, also $4 / 5$ aller Lungenerkrankungen, als gutartiger Natur bzw. als latent anzusprechen sind. Nur $5 \%$ aller Tuberkulodermkranken leiden an einer progressiven Lungentuberkulose. Diese Tatsachedeckt sich meiner Ansicht nach mitden Befunden For ch ha m mers. Dieser hat, wie wir oben schon sahen, festgestellt, daß die Sektion der Hauttuberkulösen in etwas mehr als $6 \%$ der Fälle Lungentuberkulose als Todesursache erkennen läßł. Daneben konnte er in anderen Fällen tuberkulöse Lungenprozesse als Komplikation nachweisen, die nicht unmittelbar den Tod des Kranken herbeigeführt hatten. Da ich wohl kaum fehlgehe, wenn ich die bei $5 \%$ unserer Kranken konstatierte progressive Lungentuberkulose als voraussichtliche Todesursache anspreche, ergibt sich eine weitgehende Utbereinstimmung. Die Differenz der Ergebnisse von Leloir und Grouven einerseits und mir andererseits auf eine Verschiedenheit des Materials in den untersuchten Ländern oder Landesteilen zurückzuführen, erscheint mir nicht gerechtfertigt. Denn, obwohl die beiden Autoren zu fast gleichen Ergebnissen kommen; dürften die Verhältnisse in den von ihnen untersuchten Gegenden sich wohl kaum so gleichen, wie die in den Landstrichen, die das Krankenmaterial der Bonner und der Breslauer Universitätsklinik liefern. Das Hinterland der Bo n ner Klinik umfaßt einmal die dichtbewohnten Industriezentren des Rheinlandes, andererseits neben fruchtbarem Ackerland ärmliche Gebirgsgegenden in der Eifel, dem Westerwald und Hundsrück ${ }^{4}$ ). Die in Breslau sich zur Behandlung einfindenden Kranken setzen sich aus Bewohnern der Provinzen Schlesien und Posen zusammen, daneben eine geringe Zahl aus der Provinz Brandenburg und dem früheren Russisch-Polen. Bei weitem der größte Prozentsatz der Bresla uer Lupuskranken entfällt nach der Statistik.A. Neissers5)

1) Jadassohn, 1. c. S. 505.

2) Raw, Nathan, Is Lupus caused by the bovine tuberculosis? Tuberculosis 8, 295. 1909 .

3) Forohhammer, 1. c. S. 11.

4) Grouven, 1. c. S. 161.

5) A. Neisser, Über die Bedeutung der Lupuskrankheit und die Notwendigkeit ihrer Bekämpfung. Leipzig. 1908. 
auf die Provinz Schlesien. Wir haben also auch in Bresla u Patienten aus den fruchtbaren Landstrichen der Provinz Posen, dem Industriezentrum des oberschlesischen Kohlengebietes und den Schlesien nach Südwesten begrenzenden Gebirgsstöcken. Grouvens Material setzt sich aus Kranken mit Lupus vulgaris und Skrofulodermen zusammen, und zwar so, daß die reinen Fälle von Skrofuloderm $6 \%$ der Gesamtzahl ausmachen. Bei den von mir untersuchten Patienten wurde in 7 Fällen, also $7 \%$, ein nicht mit einer anderen Form des Tuberkuloderm kombiniertes Skrofuloderm festgestellt. Also auch in der Häufigkeit des Auftretens der einzelnen Formen besteht ein gleichsinniges Verhalten.

Von den untersuchten Kranken sind 42 männlichen und 58 weiblich e $\mathrm{n}$ Geschlechts, davon leiden an einer Lungentuberkulose 8 bzw. 17. Es sind also $20 \%$ der männlichen Hauttuberkulösen gegenüber $30 \%$ der weiblichen an einer Lungentuberkulose exkrankt. Die sich daraus ergebende Tatsache der geringeren Widerstandsfähigkeit der Lungen des weiblichen Geschlechts wird noch schärfer durch die Feststellung beleuchtet, daß von den 5 progressiven Phthisen nur eine bei einem Manne, 4 dagegen bei Frauen konstatiert worden sind, also 2,5\% und $9 \%$. Aus Grouvens Statistik ergibt sich, daß von den männlichen Kranken (250) etwa $27,5 \%$, von den weiblichen (335), etwa $32 \%$ an einer Lungentuberkulose erkrankt sind; die Verschiedenheit der beiden Geschlechter in ihrem Verhalten zu einer Komplikation mit Lungentuberkulose tritt also nicht so deutlich hervor, besteht aber ebenfalls. Forchhammers Daten lassen bei 58 seiner Sektionen an Hauttuberkulösen, die eine Phthise als Todesursache ergeben, das Geschlecht bestimmen; es finden sich 13 Männer und 45 Frauen darunter, also etwa $22 \%: 78 \%$. Diese Zahlen lassen sich aber, wie schon oben ausgeführt, nur mit den analogen bei Patienten mit progressiver Phthise vergleichen. Da unter unseren 5 progressiven Lungentuberkulosen sich ein Mann und 4 Frauen befinden, lauten die entsprechenden Prozentzahlen $20: 80$, also abermals eine auffallende UUbereinstimmung der Zahlen, die durch die exakteren Methoden der Untersuchung bei Forchham mer und mir gewonnen werden. Unter den 100 Patienten unserer Klinik befinden sich $17 \mathrm{Kranke}$ im Kindesalter (bis einschließlich 15 Jahre alt), davon sind 7 Knaben und 10 Mädchen. Bei einem Mädchen besteht ein fraglich positiver Lungenbefund, sonst ist er in allen Fällen negativ.

Betrachtet man die einzelnen Formen der Hauttuberkulose in ihrem Verhalten zur Lungentuberkulose, so ist es der auf eine Körperregion lokalisierte Lupus vulgaris mit gleichzeitiger Erkrankung der Schleimhäute, der am häufigsten mit einer Lungentuberkulose kombiniert ist (35\%, davon $27 \%$ indurative, $8 \%$ progressive). Der Lupus ist dabei 
mit Ausnahme zweier Fälle, in denen die vorderen oder seitlichen Halspartien befallen sind, immer im Gesicht, und hier wieder meistens an der Nase, lokalisiert. Weniger sind die Skrofuloderme und die Tuberculosis cutis verrucosa in ihren verschiedenen Spielarten mit Lungeninfektion kombiniert, beide in rund $30 \%$. Merkwürdigerweise sind die Lungenerkrankungen bei dem multiplen disseminierten Lupus vulgaris bedeutend seltener (15\%). Am günstigsten verhält sich der Lupus vulgaris ohne gleichzeitige Schleimhauterkrankung mit 12\% Lungenkomplikationen. Auch hier ist das Gesicht die bevorzugte Körperregion, es ist in 21 von 26 Fällen erkrankt, in drei Fällen ist es der Hals und in zweien sind es die Extremitäten. Alle Iungentuberkulosen sind hier indurativer Art.

Tch komme also zu demselben Ergebnis wie Forchbammeri) auf Grund seiner Sektionsbefunde:

Die Fälle von Lupus vulgaris der Haut und der Schleim$\mathrm{h} a$ utedes Gesichts sind a $m$ häufigsten mit einertuberkulösen Infektion der Lungen (einer allgemeinen tuberkulösen Infektion nach Forchhammer) kombiniert.

Fehlt dagegen die Schleimhauterkrankung, so ist die Wahrscheinlichkeit einer Komplikation des Gesichtslupus mit einer Lungentuberkulose um ein vielfaches geringer.

2. 3. Bei der Erörterung der Frage, welchen Einfluß das Alter des an einer Hauttuberkulose Erkrankten auf die Häufigkeit einer Infektion der Lungen mit Tuberkulose hat, erkennt man bald, daß die hier untersuchte Zahl von Patienten zu klein ist, um sichere. Schlüsse daraus ziehen zu können. Schon im vierten und in den folgenden Dezennien sind die absoluten Zahlen so unbedeutend, daß eine vergleichende prozentuale Berechnung keinen Anspruch auf Zuverlässigkeit machen darí. Genau das gleiche gilt von der Untersuchung der Beziehungen zwischen dem Alter, d. h. der Dauer des Bestehens der Hauttuberkulose, und dem gleichzeitigen Vorkommen einer Lungentuberkulose. Nur, daß hier schon im dritten Dezennium die Patientenzahl sehr gering ist. Ich glaube mich daher mit einer tabellarischen Ubersicht in den Zusammenstellungen II und IIT begnügen zu dürfen. Zur schnelleren Orientierung seien die beiden entsprechenden graphischen Zeichnungen hinzugefügt. Diese geben in den breiten Säulen die absoluten Zahlenwerte der erkrankten Männer und Frauen in den verschiedenen Dezennien nach dem Alter der Kranken bzw. in halben Dezennien nach dem Alter des Tuberkuloderms an. Daneben sind, in den schmalen Säulen, die absoluten Zahlen der entsprechenden Kranken, die gleichzeitig mit einer Phthise behaftet sind, vergleichsweise niedergelegt.

1) Forchtamer, 1. c. p. 8. 
Die Lingentuberknlose als Komplikation der Tuberkuloderme.

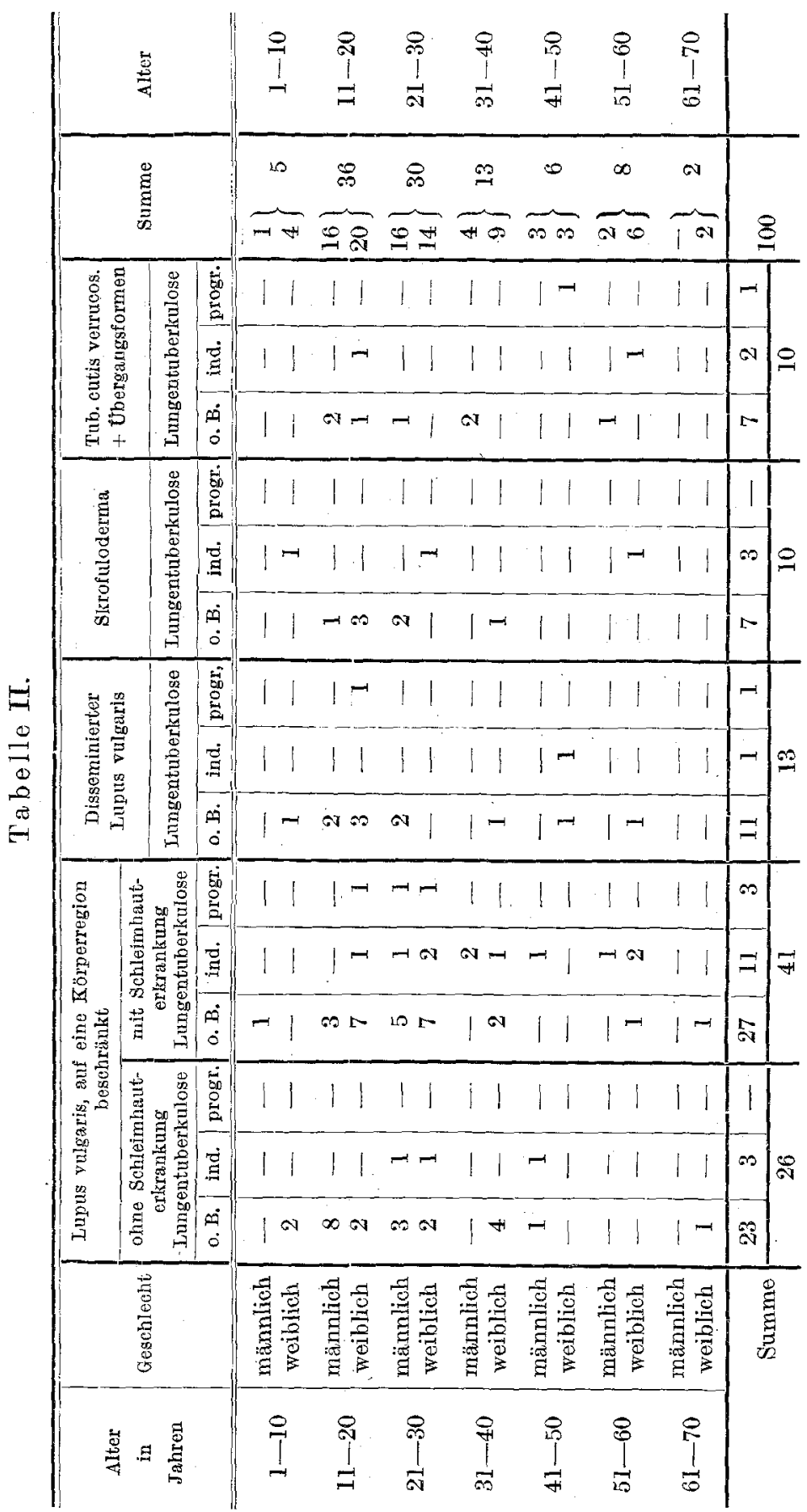




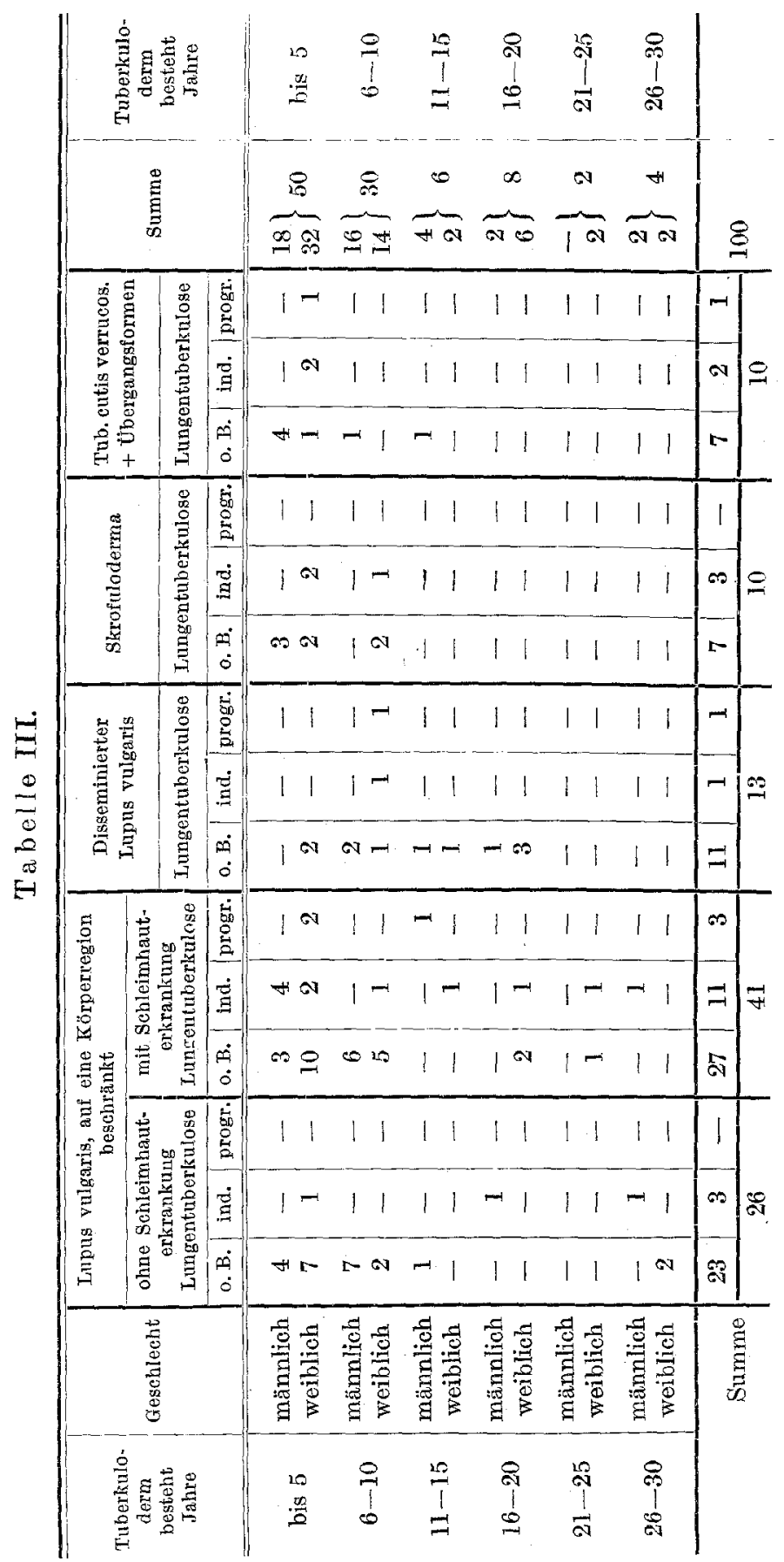


Die Lungentuberkulose als Komplikation der Tuberkuloderme.
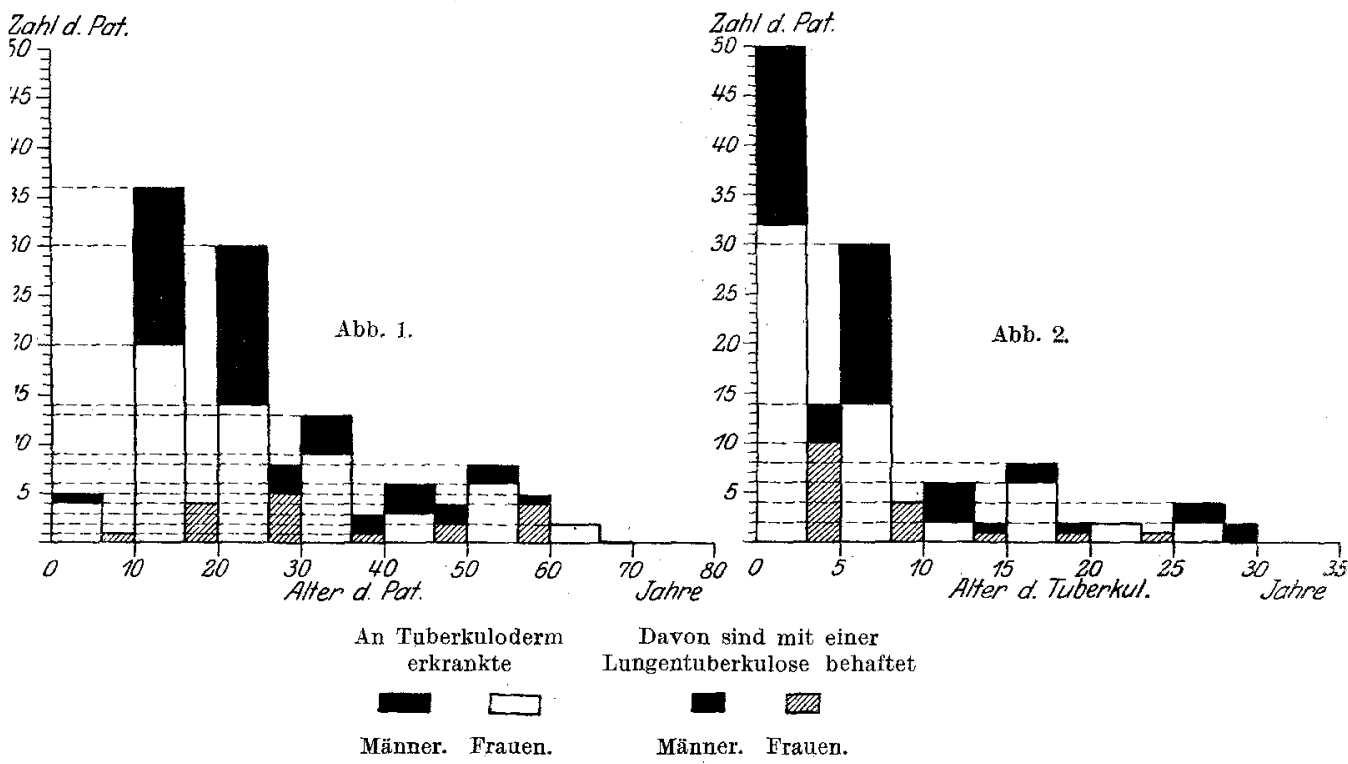

Auch die prozentuatê Berechnung der Lungentuberkulosen im Verhältnis zum Alter der Patienten, bzw. zu der Dauer des Bestehens der Hauttuberkulose dient lediglich zur Information (Tabellen II a und IIIa).

Tabelle II a

\begin{tabular}{c|c|c||c|c}
\hline \hline $\begin{array}{c}\text { Altex der } \\
\text { Patienten. }\end{array}$ & \multicolumn{3}{|c|}{ An Lungentuberkulose sind erkrankt } \\
\cline { 2 - 4 } Dezenuium & v. sämtl. Pat. & nach Grouwen & v. mänvl. Pers. & v. weibl. Pers. \\
\hline I & $20 \%$ & $25,8 \%$ & $0 \%$ & $25 \%$ \\
II & $11 \%$ & $24,5 \%$ & $0 \%$ & $20 \%$ \\
III & $26 \%$ & $36 \%$ & $19 \%$ & $35 \%$ \\
IV & $24 \%$ & $36 \%$ & $50 \%$ & $11 \%$ \\
V & $67 \%$ & $32,4 \%$ & $67 \%$ & $67 \%$ \\
VI & $63 \%$ & $38,4 \%$ & $50 \%$ & $67 \%$ \\
VII & $0 \%$ & $9 \% !$ & $0 \%$ & $0 \%$
\end{tabular}

Tabelle IIIa

\begin{tabular}{l|c|c|c}
\hline Alter des Tuberkulo- & \multicolumn{3}{|c}{ An Lungentuberkulose sind erkrankt } \\
\cline { 2 - 4 } derms & v. sämtl. Pat. & v. männl. Pat. & v. weibl. Pat. \\
\hline bis 5 Jahre inc. & $28 \%$ & $22 \%$ & $31 \%$ \\
$5-10$ Jahre & $13 \%$ & $0 \%$ & $29 \%$ \\
$11-15$ Jahre & $33 \%$ & $25 \%$ & $50 \%$ \\
$16-20$ Jahre & $25 \%$ & $50 \%$ & $17 \%$ \\
$21-25$ Jahre & $50 \%$ & - & $50 \%$ \\
$25-30$ Jahre & $50 \%$ & $100 \%$ & $0 \%$ \\
$31-35$ Jahre & - & - & -
\end{tabular}

1) Nach Jadassohn, 1. c. S. 304 .

Archiv f. Dermatologie u. Syphilis. o. Bd. 131. 


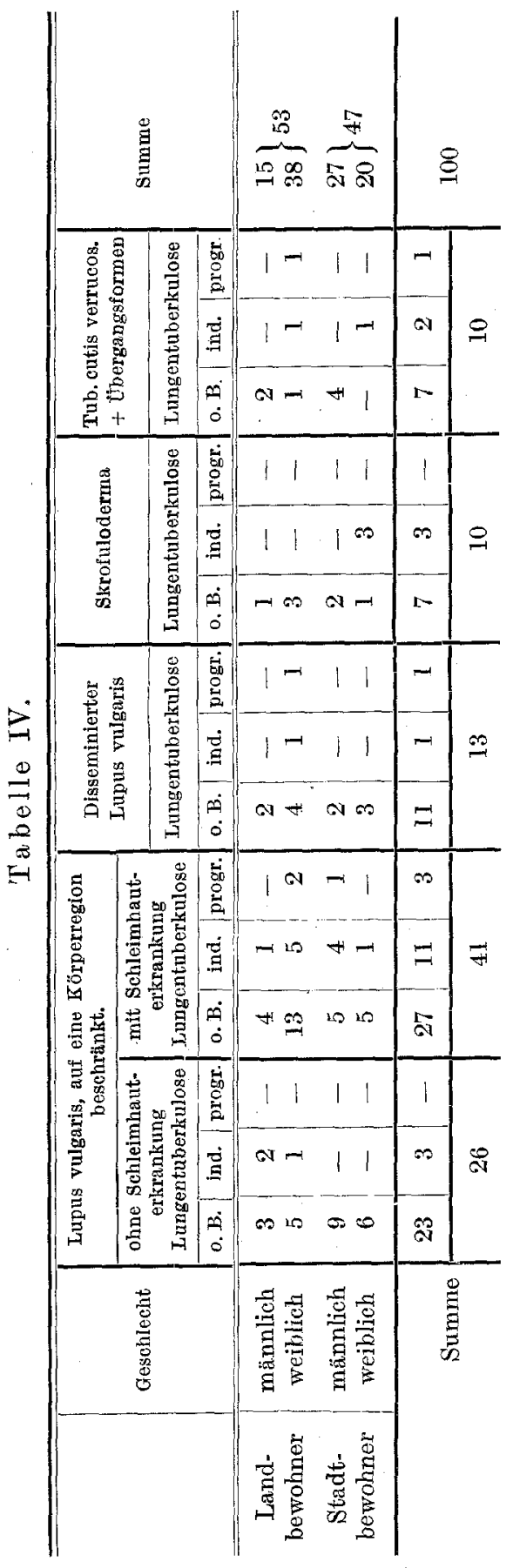

Diese Untersuchungen, die hier bei der Kleinheit des Materials nur methodologischen Wert besitzen, werden erst eine zu berechtigten Schlußfolgerungen verwertbare Grundlage abgeben, wenn sie durch anderweitiges, in gleicher Weise bearbeitetes Untersuchungsmaterial ergänzt werden. Sobald die äußeren Verhältnisse es gestatten, beabsichtige ich eine zweite Folge von hundert an Tuberkulodermen exkrankten Leuten unter denselben Gesichtspunkten zu untersuchen. Immerhin darf man wohl sagen, daß die an Hauttuberkulose leidenden Menschen mit zunehmendem Lebensalter und zunehmendem Alter des Tuberkuloderms in steigendem Maße zu einer Komplikation mit Lungentuberkulose neigen. Dies gilt jedoch nur, wenn man die Gesamtzahl der Lungentuberkulosen berücksichtigt. Dagegen scheinen die schweren Formen der Lungentuberkulose, also die progressiven, das Pubertätsalter und das dritte Dezennium zu bevorzugen; sie sind es auch, die meist in einem frühzeitigen Stadium des Tuberkuloderms in Erscheinung treten.

4. Wenn wir uns der Erörterung über die Beteiligung der Landbevölkerung und der Stadtbewohner an den tuberkulösen Lungenerkrankungen bei Hauttuberkulose zuwenden, so ergibt sich, daß die Landbevöllkerung absolut und prozentual eine größere Zahl an Phthisikern unter den an Tuberkuloderm Erkrankten aufzuweisen 
hat als die städtische. Die absoluten Zahlen verhalten sich wie $3: 2$, die prozentualen wie $29: 21$. Noch ungünstiger liegen die Verhältnisse auf dem Lande, wenn man nur die progressiven Lungentuberkulosen in Betracht zieht; das Verhältnis von Stadt und Land ist dann 4:1, bzw. $7,6 \%: 2,1 \%$. Daß die Landbevölkerung eine größere Zahl von Lupuskranken stellt, als die Stadtbewohner, ist eine Tatsache, die auch von anderen Autoren berichtet wird, so von Engelbreth ${ }^{\mathbf{1}}$ ). Dieser Autor und einzelne andere führen dies auf den Umstand zurück, daß die Tuberkuloderme auf Infektion mit dem Typus bovinus beruhen. Was aber Gilchrist ${ }^{2}$ ), Grosser ${ }^{3}$ ), Rup $\mathrm{p}^{4}$ ) u. a. auf klinischem und statistischem Wege nachzuweisen versucht haben, wird durch die Laboratoriumsuntersuchungen, abgesehen von älteren Mitteilungen in diesem Sinne (Zieler ${ }^{5}$ ), Burnet, Rothe und Bierotte, Weber und Taute), in einer Arbeit aus Finsens medizinske Lysinstitut, die demnächst im Archiv für Dermatologie und Syphilidologie erscheinen dürfte, bewiesen: daß der Typus bovinus gegenüber dem Typus humanus als Erreger des Lupus vulgaris und selbst der Tubereulosis cutis verrucosa eine untergeordnete Rolle spielt. Auch die relative Häufigkeit der Lungentuberkulose als Komplikation des Tuberkuloderms unter den Landleuten steht damit im Finklang. Dabei ist auf dem Lande eine Kombination eines Tuberkuloderms mit Lungentuberkulose bei dem weiblichen Geschlecht viermal so häufig als bei dem männlichen. Und, wie schon oben gesagt, ist die Lungentuberkulose bei der weiblichen Landbevölkerung nicht nur häufiger, sondern tritt auch in weit schwererer Form unter ihr auf. Bei der städtischen Bevölkerung ist ein Unterschied zwischen den Geschlechtern in ihrem Verhalten zur Lungentuberkulose nicht zu konstatieren. Die einzige hier festgestellte progressive Lungentuberkulose hat einen Mann befallen. Das Hauptkontingent der Phthisiker wird aber auf dem Lande wie in der Stadt von den Lupuskranken gestellt, die an einer Erkrankung der Haut und Schleimhäute des Gesichtes leiden.

\footnotetext{
1) Engelbreth, C., Ist Lupus Rindertuberkulose? Monatshefte f. prakt. Dermatol. 50, 247. 1910.

2) Gilchrist, C. T., An interesting group of cases of tuberc. infection the skin and allied cases. Amer. journ. of cutan. diseas. 5, 195. 1907.

3) Grosser, P., Über Impftuberkulose. Dermatol. Zeitschr. 14, 491. 1907.

4) Rupp, Ernst, Klinischer und statistischer Beitrag zur Ätiologie der Hauttuberkulose, insbesondere des Lupus vulgaris. Dermatol. Wochenschr. 56, 129. 1913.

5) Zieler, Karl, Hauttuberkulose und Tuberkuloide. Wiesbaden. 1914.
} 